\title{
Breves Reseñas de Investigaciones
}

Por falta de espacio y tiempo un gran número de las investigaciones, promovidas por el Programa Conjunto de Revitalización Cultural en la Costa Caribe, no pudo ser publicada. Por tal motivo hemos elaborado estas breves reseñas de algunas de ellas, para dar a conocer su existencia a los estudiosos interesados.

\section{Origen de la comunidad de Tuskru Tara Wangki Maya}

\section{Félix Antonio Ramsin Serapio}

Esta investigación relata la historia oral de la fundación de la comunidad miskita de Tuskru Tara, la cual está ubicada en el municipio de Waspam, en la ribera del río Coco, RAAN. Según las diferentes versiones recabadas por el autor, esta comunidad fue fundada en 1805 por Richard Asting y Joseph Belly, ambos originarios de Wasla, comunidad vecina de Tuskru Tara. Se cree que estos dos fundadores brindaron sus servicios en la corte del rey miskito Robert Henry Clarence, puesto que tenían múltiples conocimientos sobre caza, pesca, agricultura y sobre el clima. El nombre original con que estos señores bautizaron este lugar fue Kungkung Watla (casa del mono congo). Sin embargo, un misionero moravo de apellido Eglang, párroco de la comunidad de Wasla, la rebautizó con el nombre de Tuskru Tara en 1954. Según los ancianos de Tuskru Tara, sus antepasados afirmaban que esta comunidad comenzó siendo apenas una parcela agrícola de los pobladores de la comunidad de Wasla. En la investigación se detalla, además: diferentes versiones respecto al origen del nombre de Tuskru Tara; aspectos relacionados con las diferentes versiones existentes sobre la fundación de esta comunidad y sus causas; el nombre de los fundadores con sus familias, y costumbres y fiestas tradicionales de la comunidad.

Los principales portadores culturales en los cuales se fundamentó el autor de esta investigación fueron Prado Ingram Valendres, Edwin García, Melendrez Palacios, Orlando Masanto, Guillermo Espinosa y Lobertina Coban Rabath.

\section{Historia oral de Brown Bank}

\section{Silvia Collins}

Esta investigación es sobre Brown Bank (Loma Café), una comunidad de la cuenca de Laguna de Perlas, RAAS. Fue fundada en 1905. La primera persona que se asentó fue el Sr. Fedrick Sam Blandford, un jamaiquino que llegó hasta aquí buscando refugio. Según cuentan los portadores culturales consultados, en Jamaica, en ese tiempo, cuando alguien mataba un animal que no era suyo, el castigo era asesinar a la persona que cometió el delito. Era el caso del señor Sam Blandford. Por esta razón emigró hasta la cuenca de Laguna de Perlas donde se casó con Lucy Peralta, a nombre de la cual extendieron, en Marshall Point, el título de la propiedad ubicada en lo que actualmente se conoce como Brown Bank. Se dice en la comunidad que no extendieron el título a su nombre, porque él no quiso que el gobierno de Jamaica supiera su paradero. Sam Blandford se estableció con su esposa en Brown Bank donde procreó cuatro hijos. Desde que los primeros pobladores llegaron a esta comunidad, ésta nunca ha sido muy poblada.

Luego de los datos sobre el origen de Brown Bank, la autora describe: las primeras festividades; las primeras personas dedicadas a la salud, las enfermedades más usuales y las formas de curación; cómo se educaban los niños a partir de la fundación de la comunidad; cómo se proveían de agua y de alimentos y cómo cultivaban y pescaban; qué medios de transporte utilizaban y cuál era la forma de organización social. Finalmente da una visión actualizada de Brown Bank.

Los portadores culturales entrevistados fueron: Bernicia Collins Hebbert, Marta Sambola Collins, Withmore Sambola Martínez, Cecil Hebbert Martínez y George Collins Hebbert. 


\section{Mitos garífuna de Orinoco}

\section{Diony Zenon}

La comunidad garífuna de Orinoco se encuentra en el municipio de Laguna de Perlas, RAAS. En esta comunidad está asentado el núcleo garífuna de Nicaragua. Su población alcanza los 1,500 habitantes aproximadamente, la mayoría perteneciente a la etnia garífuna. El presente informe trata de documentar e identificar la historia oral de 14 mitos garífunas en esta comunidad, con el objetivo de revitalizar su transmisión generacional y contrarrestar con esto la disminución de la práctica oral de los mitos por parte de la juventud de Orinoco. Algo sumamente importante ya que estos mitos han determinado, de muchas maneras, el comportamiento social de estos comunitarios. A la par de la recopilación se investiga, con los portadores culturales y resto de la población entrevistada, la importancia de estos mitos para los garífunas y las medidas a tomar para su revitalización.

Los mitos recopilados son: Jack and the Lantern, Ferry Boy, Lassa Baby, The hog with the 12 pigs, The Crick Owner, Boots man, The fish owner, The abandon of Ebo Point, The tide, The spirit, Planting depend on the moon, After death you go to a place call sairy, The dead person picture, Cross the baby over the box.

Se identificaron siete portadores conocedores de los mitos garífuna en la comunidad: Mauricio González, Promecio Sambola González, Absalom Velázquez Zenón, Margarita Sambola, Agustín López, Victoria Martínez y Elma Hebbert Estrada.

\section{Diagnóstico sobre el patrimonio histórico material e inmaterial de la comunidad sumu-mayangna de Sikilta y dos barrios del municipio de Siuna, RAAN}

\section{Cristina Landaverde}

Este estudio afirma que las principales expresiones de patrimonio material cultural son la Iglesia católica y la morava, así como los teatros o cines que existieron, el gimnasio del instituto parroquial, los clubes, el cementerio, los ríos, y se hace referencia a la Bomba o Matis, como uno de los ríos más perjudicados por el despale indiscriminado. El principal sitio natural histórico de Sikilta es conocido como Kiulna. Como patrimonio inmaterial se identificaron juegos, leyendas, mitos y fiestas religiosas, casi todos de origen mestizo. El plato típico de mayor preferencia es el rondón y se retoma como una rica comida el chancho de monte cocido con leche de coco y banano. Para los mayangnas, el alimento preferido es el díkuruhna y la bebida de mayor consumo es el wabul. Se identificó a 56 gestores culturales empíricos de Siuna; de éstos, 15 ya han fallecidos, siendo los principales la señora Mercedes Gatica Salazar, los hermanos Madrigal y el señor Tipy, artista músico reconocido de la etnia creole ya fallecido.

\section{Medidas de capacidad etno-matemáticas en la comunidad sumu-mayangna de Betania}

\section{Juan Pikitle Pedro}

Así como existen medidas de capacidad occidentales como el litro, el galón, etcétera, también hay medidas o unidades de capacidad propias de la cultura sumu-mayangna. Siguiendo la actual corriente pedagógica internacional que reconoce la importancia de los conocimientos y técnicas etno-matematemáticas del propio grupo cultural al cual pertenece el educando, el autor de esta investigación espera iniciar, mediante este estudio, un proceso de introducción de medidas o unidades de capacidad ancestrales - para líquidos y sólidos- en el Sistema Educativo Autonómico Regional (SEAR). Para esto ha identificado y clasificado - en la comunidad sumu-mayangna de Betania, municipio de Rosita, RAAN- diferentes unidades de medida utilizadas ancestralmente para sus actividades productivas. Con 
esto espera contrarrestar la invasión, cultural y lingüística, occidental y los enfoques mismos de Educación Bilingüe que muchas veces ignoran que la capacidad de expresión matemática de un grupo cultural es tan propia como su capacidad de lenguaje.

Mediante una amplia consulta con los comunitarios de Betania, el autor no solo identifica y clasifica estas medidas ancestrales sino que también describe sus formas de elaboración y la posición de la comunidad ante esta problemática cultural. Además incluye algunas recomendaciones para promover estas medidas de capacidad entre la población y para introducirlas en el Sistema Educativo Autonómico Regional (SEAR). Las medidas de capacidad, típica de comunidad sumu-mayangna son: sulun, sau suba, pispis o sumah, suku, wili, sûtak, katak, baul, plapta.

\title{
Un problema de salud comunitaria: kal bubulna yamahni, la enfermedad de los malos espíritus
}

\author{
Juan Modesto Rener Martinez
}

Kal bubulna yamahin es una extraña y antigua enfermedad que han sufrido los indígenas miskitos y mayangnas, principalmente. Ataca indistintamente a hombres, mujeres y niños. El autor de este estudio la investigó en tres comunidades del territorio Mayangna Sauni As, en la RAAN, a través de personas que la han padecido y con los ditalyang (médicos tradicionales) que la han curado. En este territorio, desde hace tres años vienen sufriendo esta enfermedad. Sus síntomas iníciales son dolores de cabeza acompañados de calentura. Luego, con el avance y gravedad de la enfermedad, el que la padece ve visiones, escucha voces extrañas y hasta llega a perder su sano juicio. En casos extremos ha causado la muerte accidental de las personas. Según los curanderos, la enfermedad es causada por malos espíritus que existen en las montañas o por sirenas tiene su hábitat en los ríos. Su cura es mediante plantas medicinales, previo a la identificación de esta enfermedad, pues existen variedades de kal bubulna yamahni.

El autor, durante la investigación contó con la colaboración de Leónicia Rufus, Salvador Edwin y Manolo Palacios, todos ellos ditalyangs.

\section{Conocimientos ancestrales agrícolas practicados en cinco comunidades del Territorio Mayangna Sauni As}

Alfonso Mauricio Robins Jacobo

A través de esta investigación fueron rescatados los conocimientos ancestrales practicados en las actividades agrícolas de cinco comunidades ubicadas en la Reserva de Biosfera de Bosawas, específicamente, en el Territorio Mayangna Sauni As: Musawas, Alal, Wingpulu, Sabawas y Betlehen, consideradas como las más antiguas y donde viven los portadores de la historia y la cultura de estos pueblos. En todas ellas se investigó la relación entre las diferentes herramientas utilizadas en la actividad agrícola y las técnicas implementadas; los elementos claves de la conservación del medio ambiente y su significado en el mundo cosmogónico de la cultura mayangna, y los elementos simbólicos utilizados en estas actividades.

Entre los resultados más importantes de esta investigación está la conclusión de que, a pesar de que estas comunidades mayangnas han transitado de la recolección, la caza y la pesca, hasta la tumba, roza y quema (pasando por el sistema del boleo), es evidente que, gracias a su cultura, cosmovisión y economía, han logrado mantener una conexión muy fuerte con la existencia del bosque, que en su pasado ha tenido una función vital en su formas de vida. El Mayangna, basado en su propia forma de ver el mundo, acondiciona y acopla todos los elementos que le son de importancia para su bienestar y el cuidado del bosque. 


\title{
Alternativas que ofrece el ecoturismo comunitario en tres comunidades del Territorio Mayangna Sauni Arungka
}

\author{
Leonardo Juwith Bendlis
}

Este estudio se hizo con la finalidad de saber, en tres comunidades (Mahalwas, Ispayulilna y Mukuswas) del territorio Mayangna Sauni Arungka, el grado de conocimiento que tienen estos indígenas sobre el concepto de “ecoturismo” y las alternativas que ofrece para ellos esta modalidad turística. Los resultados reflejaron que aunque la mayoría de los ancianos entrevistados desconoce el concepto que encierra el término “ecoturismo”, sin embargo, por otro lado están convencidos de que ellos son, por naturaleza y tradición, conservacionistas de la flora y la fauna de su territorio. Han llegado a esta conclusión con la ayuda de un proyecto -Corazón Verde- que se ejecuta sobre turismo comunitario en el Territorio. A través de estos conocimientos se dan cuenta de la importancia de saber administrar y aprovechar los recursos naturales del bosque y el valor que tienen, para la explotación turística, sus conocimientos sobre la identificación de la flora y la fauna, por un lado, y por otro, los diversos e impresionantes paisajes que ofrece su territorio.

En este sentido identificaron varios sitios apropiados para desarrollar el ecoturismo comunitario: Awa Was, un sitio explotado por ellos, donde existe materia prima como oro, plata y diamante cromo; el cerro Uban, desde cuya altura se puede contemplar las tres minas: Rosita, Siuna y Bonanza; otro cerro llamado Ayan Mainh, que significa cerro mina de hierro y en cuyos alrededores existe una magnífica laguna azul; Mukuswas, un río muy caudaloso; el cerro Wala AsanWassah, un verdadero pulmón en Centro América, entre otros recursos paisajísticos.

\section{Documentación de la preparación y consumo ancestral del platillo gastronómico garífuna Bami o Ereba}

\section{Dalky Sambola}

Este estudio recopila la información sobre la preparación y consumo del platillo garífuna Bami o Ereba en la comunidad garífuna de Orinoco, municipio de Pearl Lagoon, RAAS. Quizá la propiedad más asombrosa de este platillo hecho a base de yuca sea el hecho que se conserva sin refrigeración hasta por un período de seis meses. Su consumo es un rasgo muy importante de la identidad garífuna, y uno de los incentivos para investigarlo es que ha disminuido su elaboración y consumo en la comunidad. Según la investigación, la causa principal de esto es que es "muy tediosa” su preparación (requiere diez horas de trabajo y su cocción necesita mucho fuego), además, que ahora existe mucho pan y cocinas de gas (para la elaboración del Bami es necesario un fogón). La investigación, además de enumerar los instrumentos y presentar la receta para su preparación, identifica los portadores culturales que aún lo elaboran en la comunidad: Francela Francisca Hodgson Jiménez, Margarita Sambola González, Julia Jiménez López, Elma Herbberth Estrada, Sonia Cash Martines, Aída López Hebberth, Eloisa López Hebberth, Estifania Sambola González, Alejandrina López Monroe, Amanda Sambola.

\section{Plantas medicinales en las comunidades de Wasakin, Fruta de pan y Finicia}

\section{Tomasa Gómez Mordy}

El presente trabajo investigativo aborda la medicina tradicional de la población sumu-tuahka, que habita las comunidad de Wasakin, Fruta de pan y Fenicia, en el municipio de Rosita, Región Autónoma del Atlántico Norte (RAAN). El estudio identifica las diez plantas medicinales más comunes y determina sus usos, beneficios y forma de aplicarlas. Las diez plantas medicinales son: aguacate, para la diarrea; guanábana, para los sustos y los parásitos; albahaca, para el espíritu de los muertos; culantro, para la gastritis; sandiego, para la calentura; pico de pájaro, para malestares estomacales y malos espíritus (específicamente, las sirenas), zacate limón, para la fiebre; guayaba, para la diarrea; achiote, para las inflamaciones; wana, para pescar.También identifica otros tipos de plantas que sirven para aromatizar, ornamentar, condimentar y alimentar. Finalmente presenta un listado de los portadores culturales entrevistados al respecto. 


\section{Sistema de administración de justicia tradicional en las regiones autónomas del Caribe de Nicaragua}

Efraín Benítez Miguel

Esta investigación se realizó en el territorio Mayangna Sauni Arungka (MATUMBAK), en Bonanza, RAAN, con el objetivo de analizar la aplicación de las leyes tradicionales indígenas, tomando como ejemplo tres comunidades del territorio Mayangna Sauni Arungka. Para esto analiza las legislaciones internacionales, nacionales y autónomas, incluido el Código Penal de la Mosquitia, y sus relaciones con el Derecho Consuetudinario Indígena (DCI); estudia la conformación y organización del territorio MATUMBAK, y valora su autonomía territorial y comunal. Todo esto con la finalidad de aportar la simiente de un documento sobre nuestro sistema de administración de justicia tradicional versus la justicia occidental.

Según el autor, el derecho indígena lleva una concepción jurídica diferente, ya que los principios de tolerancia y diversidad cultural, social y natural constituyen el centro de la ley y regulan la vida social con dignidad, equilibrio y en armonía con la madre naturaleza. Mientras que los principios jurídicos traídos de Europa a América se basan en la separación del hombre y la naturaleza. En este sentido analiza el grado de aplicación de las leyes tradicionales y los papeles de las principales autoridades comunales: el juez o wihta, el síndico, el Consejo de Ancianos y la Asamblea Comunal, en el marco de la administración de justicia que tiene que ver con los derechos de propiedad de la tierra y sus recursos naturales, el matrimonio, y las transgresiones de orden social, con sus debidas aplicaciones de leyes tradicionales.

El autor recopiló esta información con la ayuda de los portadores culturales del territorio MUTUMBAK: Benitez Oniel, C. Wassah, Lorenzo Lopez y Delicia Castro.

\section{Actos culturales religiosos del pueblo ulwa Storbey Simon Watson}

Este estudio sobre los actos religiosos del pueblo ulwa de Karawala, en la RAAS, tiene como objetivo documentar su aporte al desarrollo comunal desde el punto de vista cultural. Estos actos culturales, conocidos por la comunidad como pulanka nani (los juegos) generan armonía entre los pueblos indígenas y afrodescendientes que conforman esta comunidad. Su origen es religioso, sin embargo han derivado en enfoques sobre la realidad social. Es una tradición que ha tenido mucha importancia en la vida cotidiana de los pobladores de Karawala, pues genera reflexión sobre la realidad. En los últimos diez años, esta actividad ha formado parte de los esfuerzos por la revitalización de la lengua ulwa; además orienta y promueve valores morales y el desarrollo de capacidades artísticas de los jóvenes y contribuyen a generar ingresos en la comunidad. Para los comunitarios han sido los eventos de entretenimiento mejor organizados, además son económicos y de fácil acceso. Potencialmente se espera que en un futuro este arte se desarrolle como un recurso para toda la comunidad y como un medio de promoción del pueblo ulwa en Karawala como destino turístico cultural.

Los portadores culturales de este teatro de la comunidad ulwa son: Sara Catral Crimins, Bianey Diaz Abraham, José I. Simons Chavarría, Duarte Palmiston, Roy Salazar, Josefina Davis, Haglan Santiago, Jarquín Valeriano, Dexter Levy Hall, Zeneyda Cristian, Derlin Shogreen, Paterson, Floyd Richard, Carolina Chavarría, Ignacio Simons Walter, Alicia Shogreen Paterson, Arnoldo Simón Williams, Benicia Rodríguez, Orin Palmiston, Baptis Crimins, Chester Lam Palmiston, Claratina Davis, Darna Wilson Crimins, Delrey Simón Chavarría, Edwin Campbell Knigth, Evarista Valeriano Simón, Evelyn Martínez, Feliciano Mails Crimins, Gregory Palmiston Wilson, Irma Simons Hodgson, Jessica Francis, Julia Díaz Simon, Lidia Pondler, Liroy Simón, Alberto Santiago, Marció García Davis, Marilee Abraham Palmiston, Mayra Morales Wilson, Omar Ruiz Díaz, Raynaldo Simon Ch, Ronald Ingram Díaz, Roy Salazar Gómez, Selsa William Crimins, Sherman Flores, Rufos Simon, Suleyka Ingram Price, Wilbort Bent Davis, Yahoska Díaz Abraham 